\title{
NORM-CONTROLLED INVERSION IN SMOOTH BANACH ALGEBRAS, I
}

\author{
KARLHEINZ GRÖCHENIG AND ANDREAS KLOTZ
}

\begin{abstract}
Every differential subalgebra of a unital $C^{*}$-algebra is spectrally invariant. We derive a quantitative version of this well-known fact and show that a minimal amount of smoothness, as given by a differential norm, already implies norm control. We obtain an explicit estimate for the differential norm of an invertible element $a$. This estimate depends only on the condition number of $a$ and the ratio of two norms.
\end{abstract}

\section{INTRODUCTION}

In many contexts one encounters the problem of inversion of smooth elements in a Banach algebra. Often the smoothness is preserved under inversion, and one is challenged to control the smoothness norm of the inverse. This is the quantitative problem of norm-controlled inversion.

The standard example of norm-controlled inversion occurs already in calculus under the name of the quotient rule. Let $C^{1}(\mathbb{T})$ be the algebra of continuously differentiable functions on the torus $\mathbb{T}$ and $C(\mathbb{T})$ be the algebra of all continuous functions on the torus with the norms $\|f\|_{C}=\|f\|_{\infty}=\max _{t \in \mathbb{T}}|f(t)|$ and $\|f\|_{C^{1}}=\|f\|_{\infty}+\left\|f^{\prime}\right\|_{\infty}$. The quotient rule $(1 / f)^{\prime}=-f^{\prime} / f^{2}$ leads to an obvious estimate for the $C^{1}$-norm of $1 / f$, namely,

$$
\left\|\frac{1}{f}\right\|_{C^{1}} \leq\left\|\frac{1}{f}\right\|_{\infty}+\left\|f^{\prime}\right\|_{\infty}\left\|\frac{1}{f}\right\|_{\infty}^{2}=\left(\|f\|_{C^{1}}\left\|\frac{1}{f}\right\|_{C}+1\right)\left\|\frac{1}{f}\right\|_{C} .
$$

In other words, we can control the norm of the inverse of $f$ in the subalgebra $C^{1}(\mathbb{T})$ by the norm of $f$ in $C^{1}(\mathbb{T})$ and the norm of $1 / f$ in $C(\mathbb{T})$. This is the phenomenon of norm control in a nutshell. Can we obtain quantiative estimates for the norm of the inverse in a subalgebra, when we have access to information about the inverse in an ambient algebra? Usually invertibility and norm bounds are easier to obtain in the large algebra.

In applied mathematics this problem is underlying the regularity of solutions of linear systems in infinite-dimensional spaces. If a matrix possesses off-diagonal decay and is invertible on $\ell^{2}$, then its inverse possesses the same quality of off-diagonal decay [3, $4,20,23]$. As a consequence the solution of the linear equation $A x=b$ with an input vector $b$ of a certain decay possesses a solution $x$ with the same decay. This principle has numerous and often fundamental applications in numerical analysis $[9,21,27]$, the theory of pseudodifferential operators [16,32], in frame theory [2,15], in time-frequency analysis [19], and in sampling theory [15,35].

2000 Mathematics Subject Classification. 41A65,46H30,47L99,47A60.

Key words and phrases. Inverse-closed Banach algebra, norm-control, differential algebra, approximation space, asymptotic analysis.

Both authors were supported by the project P22746-N13 of the Austrian Science Foundation (FWF). 
In non-commutative geometry smooth subalgebras and their spectral invariance are an important technical tool for the computation of the $K$-group of an algebra. The density theorem asserts that the $K$-groups of a smooth subalgebra coincide with the $K$-groups of the original $C^{*}$-algebra [6].

A general theory of abstract smoothness and an axiomatic construction of inverseclosed subalgebras was developed in $[17,25]$. With a minimum amount of natural structures on a given Banach algebra, namely unbounded derivations or commutative automorphism groups, one can construct subalgebras of smooth elements where the smoothness mimicks the Besov regularity or the Hölder-Lipschitz continuity of functions on $\mathbb{R}^{d}$. This theory establishes a new link between methods of approximation theory and the theory of operator algebras. So far, this construction of smooth subalgebras and their inverse-closed is a purely qualitative theory: if an element is smooth, then its inverse possesses the same quality of smoothness.

The obvious next step is to develop quantitative statements. What is the "size" (norm) of the inverse element in the smooth subalgebra? This is the problem of norm-controlled inversion. The quantification is of utmost importance in applied or numerical constructions, where the control of the constants decides the success or failure of a method. Naturally, these problems have been investigated first in the context of the off-diagonal decay of infinite matrices, see [3]. It has been known for a long time that an invertible matrix with exponential decay off the diagonal has an inverse again with exponential off-diagonal decay [10], the question of the precise constants involved turned out to be fundamental for many applications. For polynomial off-diagonal decay Baskakov has studied a subtle version of norm-control [4].

The term "norm control" is due to Nikolskii [28]. He studied "the invisible spectrum" in the algebra of absolutely convergent Fourier series and obtained estimates for the norms of inverses in this algebra. Interestingly enough, the Fourier algebra is one of few examples so far, for which the lack of norm control is known. As soon as one passes to absolutely convergent weighted Fourier series, one can prove norm control [13,14].

In an abstract context one may say that smoothness implies inverse-closedness. The topic of this paper is the much stronger statement that smoothness implies norm control.

In this paper and a subsequent paper we will study the concept of norm control in smooth subalgebras in a systematic manner. (a) What is smoothness in an abstract Banach algebra? (b) Which forms of smoothness are preserved under inversion? (c) Which forms of smoothness admit norm-control? The first two questions were studied and answered (at least partially) in [17], the third question will be the central topic of this paper.

In our main result we derive explicit norm-controlled inversion for subalgebras defined by an extremely weak condition of smoothness that is expressed by a differential (semi) norm. This type of smoothness has been used heavily in operator theory and non-commutative geometry [7,24,31] and arises naturally in approximation theory [11].

Theorem 1.1. Assume that $\mathcal{B}$ is a $C^{*}$-algebra and $\mathcal{A} \subseteq \mathcal{B}$ is a *-subalgebra with a common unit and a norm satisfying the inequality

$$
\|a b\|_{\mathcal{A}} \leq C\left(\|a\|_{\mathcal{A}}\|b\|_{\mathcal{B}}+\|b\|_{\mathcal{A}}\|a\|_{\mathcal{B}}\right)
$$


for all $a, b \in \mathcal{A}$. Then there exist constants $\gamma_{j}$ depending only the structure constant $C$, such that the following norm control estimates hold:

If $a \in \mathcal{A}$ and $a$ is invertible in $\mathcal{B}$ with condition number $\kappa(a)=\|a\|_{\mathcal{B}}\left\|a^{-1}\right\|_{\mathcal{B}} \geq 5$, then

$$
\left\|a^{-1}\right\|_{\mathcal{A}} \leq \max \left\{\gamma_{1} \frac{\|a\|_{\mathcal{A}}}{\|a\|_{\mathcal{B}}^{2}} e^{\gamma_{2} \ln ^{2} \kappa(a)}, \gamma_{3} \frac{\|a\|_{\mathcal{A}}}{\|a\|_{\mathcal{B}}^{2}} e^{\gamma_{4} \ln ^{2}\left(\gamma_{5} \frac{\|a\|_{\mathcal{A}}^{2}}{\|a\|_{\mathcal{B}}^{2}}\right)}\right\}
$$

This result requires several commments.

(i) In Theorem 4.1 we will derive explicit expressions for the constants $\gamma_{j}$.

(ii) Observe in particular that for $a \in \mathcal{A}$ and $a$ invertible in $\mathcal{B}$, the inverse $a^{-1}$ is already in $\mathcal{A}$. We say that $\mathcal{A}$ is inverse-closed in $\mathcal{B}$.

(iii) Though Theorem 1.1 is a result in operator theory, the proof requires methods from approximation theory and the asymptotics of certain special functions.

(iv) The case distinction in (3) leads to an important insight: the norm of $a^{-1}$ in $\mathcal{A}$ does not only depend on the condition number $\kappa(a)$ in $\mathcal{B}$ and $\|a\|_{\mathcal{A}}$, as one might have expected from Nikolski's work [28], but also on the embedding ratio $\|a\|_{\mathcal{A}} /\|a\|_{\mathcal{B}}$. This phenomenon occurs already in the commutative case for $C^{1}(\mathbb{T})$.

(v) A norm on a subalgebra satisfying (2) is called a differential norm. This property can be considered a weak form of smoothness in $\mathcal{B}$. In [31] Rieffel defined a strong Leibniz norm as a differential (semi)norm satisfying the norm control $\left\|a^{-1}\right\|_{\mathcal{A}} \leq$ $C\|a\|_{\mathcal{A}}\left\|a^{-1}\right\|_{\mathcal{B}}^{2}$ and asked whether every differential seminorm is a strong Leibniz norm. The example of $n$-times continuously differentiable functions on the torus $C^{n}(\mathbb{T})$ for $n>1$ shows that this guess cannot be true in general, nevertheless Theorem 1.1 states that a differential (semi)norm admits norm control in a weaker sense. For applications in operator theory and non-commutative analysis, e.g. for quantitative versions of the holomorphic functional calculus, Theorem 1.1 might be strong enough.

(vi) Theorem 1.1 applies in particular to approximation algebras; these are subalgebras of a given algebra that are defined by the approximation properties with respect to a socalled approximation scheme or a filtration of subalgebras.

(vii) Note that (3) implies that $\left\|a^{-1}\right\|_{\mathcal{A}} \leq \mathcal{O}\left(\kappa(a)^{C \ln \kappa(a)}\right)$ for $\kappa \rightarrow \infty$. This means that $\left\|a^{-1}\right\|_{\mathcal{A}}$, as a function of the condition number, grows faster than every polynomial. In the sequel [18] to this paper we will develop results for stronger concepts of smoothness, namely for subalgebras of a given Banach algebra with smoothness of Besov-HölderLipschitz type of $[17,25]$. These Banach algebras admit a generalized quotient rule, and we will prove much stronger statements about norm-control with polynomial growth $\left\|a^{-1}\right\|_{\mathcal{A}} \leq \mathcal{O}\left(\kappa(a)^{N}\right)$ in the style of (1). Since the methods are completely different, we prefer to split our study of norm control into two parts.

The paper is organized as follows: In Section 2 we explain the abstract concept of norm control and give some equivalent definitions. In Section 3 we prove that Banach algebras with a differential norm admit norm-controlled inversion. Surprisingly, a standard construction of approximation theory always yields Banach algebras that admit norm-controlled inversion. In Section 4 we derive the asymptotic estimate for the norm-controlling function of Theorem 1.1. In the last section we will briefly discuss other possible concepts of norm control and the failure of norm-controlled inversion. At 
this time the main example without norm control is the pair $(\mathcal{A}(\mathbb{T}), C(\mathbb{T}))$ of absolutely convergent Fourier series and of continuous functions on the torus [28].

\section{WhAT IS NORM-CONTROLLED INVERSION?}

In this section we will explore some facets of the abstract notion of norm control.

Definition 2.1. Let $\mathcal{A} \subseteq \mathcal{B}$ be Banach algebras with common unit.

(i) We say that $\mathcal{A}$ is inverse-closed in $\mathcal{B}$, if every element $a \in \mathcal{A}$ that is invertible in $\mathcal{B}$ is already invertible in $\mathcal{A}$, in short, $a \in \mathcal{A}, a^{-1} \in \mathcal{B} \Rightarrow a^{-1} \in \mathcal{A}$.

(ii) We say that $\mathcal{A}$ admits norm-controlled inversion in $\mathcal{B}$, if there is a function $h$ : $(0, \infty)^{2} \rightarrow(0, \infty)$ that satisfies

$$
\left\|a^{-1}\right\|_{\mathcal{A}} \leq h\left(\|a\|_{\mathcal{A}},\left\|a^{-1}\right\|_{\mathcal{B}}\right) .
$$

Clearly, norm control is a stronger property than inverse-closedness. If $\mathcal{A}$ admits norm control in $\mathcal{B}$, then, in particular, $\mathcal{A}$ is inverse-closed in $\mathcal{B}$. Inverse-closedness is a qualitative property, norm control is a quantitative property. The function $h$ provides an estimate for the norm of $a^{-1}$ in the smaller algebra as a function of the norm of $a$ in the small algebra and the norm of $a^{-1}$ in the large algebra.

The control function $h$ in Definition 2.1 is not unique. To obtain a well-defined control function, we use a slightly different definition of norm control.

Lemma 2.2. Let $\mathcal{A} \subseteq \mathcal{B}$ be Banach algebras with common unit element. Then $\mathcal{A}$ admits norm control, if and only if there exists a function $\phi:(0,1) \rightarrow(0, \infty)$, such that, for $a \in \mathcal{A}$,

$$
\|a\|_{\mathcal{A}} \leq 1 \quad \text { and } \quad\left\|a^{-1}\right\|_{\mathcal{B}} \leq 1 / \delta
$$

implies that $a^{-1} \in \mathcal{A}$ and

$$
\left\|a^{-1}\right\|_{\mathcal{A}} \leq \phi(\delta)
$$

Explicitly, $\phi(\delta)$ can be chosen to be

$$
\phi(\delta)=\sup \left\{\left\|a^{-1}\right\|_{\mathcal{A}}:\|a\|_{\mathcal{A}} \leq 1,\left\|a^{-1}\right\|_{\mathcal{B}} \leq 1 / \delta\right\} .
$$

The norm control function $h$ is then

$$
h\left(\|a\|_{\mathcal{A}},\left\|a^{-1}\right\|_{\mathcal{B}}\right)=\frac{1}{\|a\|_{\mathcal{A}}} \phi\left(\frac{1}{\|a\|_{\mathcal{A}}\left\|a^{-1}\right\|_{\mathcal{B}}}\right) .
$$

Proof. Given $a \in \mathcal{A}$ with inverse $a^{-1} \in \mathcal{B}$, we set $b=\frac{a}{\|a\|_{\mathcal{A}}}$. Then $\|b\|_{\mathcal{A}}=1$ and $\left\|b^{-1}\right\|_{\mathcal{B}}=\|a\|_{\mathcal{A}}\left\|a^{-1}\right\|_{\mathcal{B}}=1 / \delta$. Then by definition of $\phi$ we have

$$
\|a\|_{\mathcal{A}}\left\|a^{-1}\right\|_{\mathcal{A}}=\left\|b^{-1}\right\|_{\mathcal{A}} \leq \phi\left(\frac{1}{\delta}\right)=\phi\left(\frac{1}{\|a\|_{\mathcal{A}}\left\|a^{-1}\right\|_{\mathcal{B}}}\right),
$$

and this is (6).

The definition of norm control is adapted from Nikolski [28]. He studied inversion in commutative Banach algebras $\mathcal{A}$ embedded in $C(X)$, the space of continuous functions on some compact set $X$. Then $\mathcal{A}$ is called $\delta$-visible for fixed $\delta>0$, if there is a constant $\phi(\delta)>0$, called the visibility constant, such that $\|f\|_{\mathcal{A}} \leq 1$ and $|f(x)| \geq \delta$ for all $x \in X$ 
implies that $\left\|f^{-1}\right\|_{\mathcal{A}} \leq \phi(\delta)$. Nikolskii determined the visibility constants explicitly for the algebra of absolutely convergent Fourier series $\mathcal{A}(\mathbb{T})$. The visibility constants are $\phi(\delta)=\left(2 \delta^{2}-1\right)^{-1}$ for $\delta>1 / \sqrt{2}$ and $\phi(\delta)=\infty$ for $\delta \leq 1 / 2$, and seem to be unknown for $1 / 2 \leq \delta \leq 1 / \sqrt{2}$. Since Definition 2.1 requires $\phi(\delta)$ to exist for every $\delta>0$, the Fourier algebra does not admit norm-controlled inversion. Related concepts were also studied in $[5,30]$.

The following lemma contains several equivalent expressions for Nikolski's visibility constant.

\section{Lemma 2.3.}

$$
\begin{aligned}
\phi(\delta) & =\sup \left\{\left\|a^{-1}\right\|_{\mathcal{A}}:\|a\|_{\mathcal{A}} \leq 1,\left\|a^{-1}\right\|_{\mathcal{B}}=1 / \delta\right\} \\
& =\sup \left\{\left\|a^{-1}\right\|_{\mathcal{A}}:\|a\|_{\mathcal{A}}=1,\left\|a^{-1}\right\|_{\mathcal{B}} \leq 1 / \delta\right\} \\
& =\sup \left\{\|a\|_{\mathcal{A}}\left\|a^{-1}\right\|_{\mathcal{A}}:\|a\|_{\mathcal{A}}\left\|a^{-1}\right\|_{\mathcal{B}} \leq 1 / \delta\right\}
\end{aligned}
$$

Proof. Set $A_{\delta}=\left\{a \in \mathcal{A}:\|a\|_{\mathcal{A}} \leq 1,\left\|a^{-1}\right\|_{\mathcal{B}}=1 / \delta\right\}$. Obviously,

$$
\sup \left\{\left\|a^{-1}\right\|_{\mathcal{A}}: a \in A_{\delta}\right\} \leq \phi(\delta) .
$$

For the reverse inequality choose a sequence $a_{n} \in \mathcal{A}$, such that

$$
\left\|a_{n}\right\|_{\mathcal{B}} \leq 1 / \delta, \quad\left\|a_{n}\right\|_{\mathcal{A}} \leq 1 \quad \text { and } \lim _{n}\left\|a_{n}^{-1}\right\|_{\mathcal{A}}=\phi(\delta) .
$$

Set $\lambda_{n}=\delta\left\|a_{n}^{-1}\right\|_{\mathcal{B}}^{-1} \geq 1$ and $b_{n}=\lambda_{n}^{-1} a_{n}$. Then $b_{n} \in A_{\delta}$, because $\left\|b_{n}\right\|_{\mathcal{A}} \leq\left\|a_{n}\right\|_{\mathcal{A}} \leq 1$, $\left\|b_{n}^{-1}\right\|_{\mathcal{B}}=\lambda_{n}\left\|a_{n}^{-1}\right\|_{\mathcal{B}}=1 / \delta$. Furthermore,

$$
\begin{aligned}
\sup \left\{\left\|a^{-1}\right\|_{\mathcal{A}}: a \in A_{\delta}\right\} & \geq \liminf _{n \rightarrow \infty}\left\|b_{n}^{-1}\right\|_{\mathcal{A}} \\
& =\liminf _{n \rightarrow \infty} \lambda_{n}\left\|a_{n}^{-1}\right\|_{\mathcal{A}} \geq \lim _{n \rightarrow \infty}\left\|a_{n}^{-1}\right\|_{\mathcal{A}}=\phi(\delta) .
\end{aligned}
$$

The second equality is proved with a similar argument. Set $B_{\delta}=\left\{a \in \mathcal{A}:\|a\|_{\mathcal{A}}=\right.$ $\left.1,\left\|a^{-1}\right\|_{\mathcal{B}} \leq 1 / \delta\right\}$. Then $\sup \left\{\left\|a^{-1}\right\|_{\mathcal{A}}: a \in B_{\delta}\right\} \leq \phi(\delta)$. Using again the sequence $a_{n}$ defined above, we set $\tilde{a}_{n}=\mu_{n} a_{n}$, where $\mu_{n}=\delta\left\|a_{n}^{-1}\right\|_{\mathcal{B}} \leq 1$. By construction, $\left\|\tilde{a}_{n}^{-1}\right\|_{\mathcal{B}}=$ $1 / \delta,\left\|\tilde{a}_{n}\right\|_{\mathcal{A}} \leq 1$, and $\left\|\tilde{a}_{n}^{-1}\right\|_{\mathcal{A}} \geq\left\|a_{n}^{-1}\right\|_{\mathcal{A}}$, which implies $\lim _{n \rightarrow \infty}\left\|\tilde{a}_{n}^{-1}\right\|_{\mathcal{A}}=\phi(\delta)$.

The last equality is a just a reformulation of the second line.

Example 2.4. If $\mathcal{B}$ is a $C^{*}$-algebra, then the parameter $\delta$ can be interpreted as the smallest singular value of an element $a \in \mathcal{B}$. For instance, a Banach algebra $\mathcal{A} \subseteq \mathcal{B}\left(\ell^{2}\right)$ admits norm-controlled inversion in $\mathcal{B}\left(\ell^{2}\right)$, if every operator $A \in \mathcal{A}$ that is onto and satisfies $\|A\|_{\mathcal{A}} \leq 1$ and $\|A c\|_{2} \geq \delta\|c\|_{2}$ for all $c \in \ell^{2}$, satisfies also $\left\|A^{-1}\right\|_{\mathcal{A}} \leq \phi(\delta)$.

Pseudospectrum. Recall that the $\delta$-pseudospectrum $\sigma_{\delta}^{\mathcal{B}}(a)$ of an element $a$ in a unital Banach algebra $\mathcal{B}$ is

$$
\sigma_{\delta}^{\mathcal{B}}(a)=\left\{\lambda \in \mathbb{C}:\left\|(\lambda-a)^{-1}\right\|_{\mathcal{B}}>1 / \delta\right\} \cup \sigma^{\mathcal{B}}(a) .
$$

Expressing the definition of $\phi$ from (5) in terms of the pseudospectrum, we obtain the following reformulation of norm-controlled inversion. 
Lemma 2.5. A Banach algebra $\mathcal{A} \subseteq \mathcal{B}$ admits norm-controlled inversion if and only if there is a function $\phi$ such that for all $\delta \in(0,1)$ and all $a \in \mathcal{A}$ with $\|a\|_{\mathcal{A}}=1$

$$
0 \notin \sigma_{\delta}^{\mathcal{B}}(a) \quad \text { implies that } \quad 0 \notin \sigma_{1 / \phi(\delta)}^{\mathcal{A}}(a) \text {. }
$$

\section{NORM-CONTROLled InVERSION In DifFERENTIAL SUbALGEBRAS}

3.1. Differential Seminorms. A general framework for describing smoothness in abstract Banach algebras was introduced in an influential paper of Blackadar and Cuntz [7]. Assume that $\mathcal{A} \subseteq \mathcal{B}$ are two Banach algebras with a common unit element. A differential norm (of order 1) is a norm on $\mathcal{A}$ that satisfies

$$
\|a b\|_{\mathcal{A}} \leq C\left(\|a\|_{\mathcal{A}}\|b\|_{\mathcal{B}}+\|b\|_{\mathcal{A}}\|a\|_{\mathcal{B}}\right)
$$

for all $a, b \in \mathcal{A}$. In this case we call $\mathcal{A}$ a differential subalgebra of $\mathcal{B}$.

Differential seminorms formalize a very general concept of smoothness. Similar concepts were introduced by Kissin and Shulman [24] and Rieffel [31]. A norm satisfying (9) is also referred to as a Leibniz norm on $\mathcal{A}$.

Let us recall a simple property of the constant occuring in differential norms. We tacitly assume that the norms satisfy $\|e\|_{\mathcal{A}}=\|e\|_{\mathcal{B}}=1$.

Lemma 3.1. Assume that $\mathcal{A} \subseteq \mathcal{B}$ are two Banach algebras with a common unit element and let $\|\cdot\|_{\mathcal{A}}$ a differential norm on $\mathcal{A}$. Then either the norms $\|\cdot\|_{\mathcal{A}}$ and $\|\cdot\|_{\mathcal{B}}$ are equivalent (and $\mathcal{A}$ is a closed subalgebra of $\mathcal{B}$ ) or the structure constant $C$ in (9) is at least 1.

Proof. Equation (9) with $b=e$ says that

$$
\|a e\|_{\mathcal{A}} \leq C\left(\|a\|_{\mathcal{A}}\|e\|_{\mathcal{B}}+\|e\|_{\mathcal{A}}\|a\|_{\mathcal{B}}\right)=C\left(\|a\|_{\mathcal{A}}+\|a\|_{\mathcal{B}}\right) .
$$

Consequently,

$$
\frac{\|a\|_{\mathcal{A}}}{\|a\|_{\mathcal{A}}}=1 \leq C\left(1+\frac{\|a\|_{\mathcal{B}}}{\|a\|_{\mathcal{A}}}\right) .
$$

If $\|\cdot\|_{\mathcal{A}}$ is not equivalent to $\|\cdot\|_{\mathcal{B}}$, then $\inf _{a \in \mathcal{A}} \frac{\|a\|_{\mathcal{B}}}{\|a\|_{\mathcal{A}}}=0$ and thus

$$
1 \leq \inf C\left(1+\frac{\|a\|_{\mathcal{B}}}{\|a\|_{\mathcal{A}}}\right)=C
$$

In most applications the ambient algebra $\mathcal{B}$ is a $C^{*}$-algebra. In this case the constant for the embedding $\mathcal{A} \subseteq \mathcal{B}$ is also 1, i.e., $\|a\|_{\mathcal{B}} \leq\|a\|_{\mathcal{A}}$ for all $a \in \mathcal{A}$.

As a warm-up we repeat Brandenburg's trick which shows that a differential subalgebra is inverse-closed. For this property we only need that $\mathcal{B}$ is a symmetric Banach algebra. This means that $\sigma_{\mathcal{B}}\left(a^{*} a\right) \subseteq[0, \infty)$ for all $a \in \mathcal{B}$.

Lemma 3.2 ( $[7,8,17,24])$. If $\mathcal{B}$ is a symmetric unital Banach algebra and $\mathcal{A} \subseteq \mathcal{B}$ is a differential subalgebra with the same unit, then $\mathcal{A}$ is inverse-closed in $\mathcal{B}$. 
Proof. Applying (9) with $a=b=c^{n}$ yields

$$
\left\|c^{2 n}\right\|_{\mathcal{A}} \leq 2 C\left\|c^{n}\right\|_{\mathcal{A}}\left\|c^{n}\right\|_{\mathcal{B}}
$$

Taking $n$-th roots and the limit $n \rightarrow \infty$ yields the inequality of spectral radii $\rho_{\mathcal{A}}(c) \leq$ $\rho_{\mathcal{B}}(c)$ for all $c \in \mathcal{A}$. Since the reverse inequality is always true for $\mathcal{A} \subseteq \mathcal{B}$, we obtain the equality of spectral radii. By a Lemma of Hulanicki [22] $\mathcal{A}$ is inverse-closed in $\mathcal{B}$.

The main step (10) above can be shaped into a quantitative statement. We obtain the result that a differential subalgebra admits norm-controlled inversion. This is rather remarkable, because the condition of a differential semi-norm is an extremely weak assumption.

Theorem 3.3. If $\mathcal{A}$ is a differential *-subalgebra of a $C^{*}$-algebra $\mathcal{B}$, then $\mathcal{A}$ admits norm-controlled inversion in $\mathcal{B}$. For the norm of the inverse in $\mathcal{A}$ we obtain

$$
\left\|a^{-1}\right\|_{\mathcal{A}} \leq \frac{\|a\|_{\mathcal{A}}}{\|a\|_{\mathcal{B}}^{2}} \prod_{k=0}^{\infty}\left(1+2 \frac{\|a\|_{\mathcal{A}}^{2}}{\|a\|_{\mathcal{B}}^{2}}(2 C)^{k}\left(1-\frac{1}{\|a\|_{\mathcal{B}}^{2}\left\|a^{-1}\right\|_{\mathcal{B}}^{2}}\right)^{2^{k}-1}\right)
$$

where $C$ is the constant of (9).

Proof. Equation (9) implies that, for arbitrary $c \in \mathcal{A}$ and $k \in \mathbb{N}$, we obtain

$$
\left\|c^{2^{k}}\right\|_{\mathcal{A}} \leq 2 C\left\|c^{2^{k-1}}\right\|\left\|_{\mathcal{A}}\right\| c^{2^{k-1}}\left\|_{\mathcal{B}} \leq 2 C\right\| c^{2^{k}-1}\left\|_{\mathcal{A}}\right\| c \|_{\mathcal{B}}^{2^{k}-1}
$$

If we set

$$
\beta_{n}=\beta_{n}(c)=\left\|c^{n}\right\|_{\mathcal{A}}\|c\|_{\mathcal{B}}^{-n},
$$

then $\beta_{n}$ is submultiplicative, and (12) says that $\beta_{2^{k}} \leq 2 C \beta_{2^{k-1}}$. Induction on $k$ implies that

$$
\beta_{2^{k}} \leq(2 C)^{k} \beta_{1}
$$

Writing this inequality as an inequality for the norms of $c^{2^{k}}$, we obtain that

$$
\left\|c^{2^{k}}\right\|_{\mathcal{A}} \leq \frac{\|c\|_{\mathcal{A}}}{\|c\|_{\mathcal{B}}}(2 C)^{k}\|c\|_{\mathcal{B}}^{2^{k}}
$$

Now let $n=\sum_{k=0}^{\infty} \varepsilon_{k} 2^{k}$ be the dyadic expansion of $n$ with digits $\varepsilon_{k} \in\{0,1\}$. Let $\mathcal{F}$ be the set of all sequences $\varepsilon=\left(\varepsilon_{k}\right) \in\{0,1\}^{\mathbb{N}}$ that contain only finitely many 1 's, then the dyadic expansion is a bijection from $\mathbb{N}$ onto $\mathcal{F}$. We now use the submultiplicativity of $\beta_{n}$ and this bijection to derive an estimate of $\left\|c^{n}\right\|_{\mathcal{A}}$ for arbitrary $n$, namely

$$
\begin{aligned}
\left\|c^{n}\right\|_{\mathcal{A}} & =\left\|\prod_{k=0}^{\infty} c^{2^{k} \varepsilon_{k}}\right\|_{\mathcal{A}} \leq \prod_{k=0}^{\infty}\left\|c^{2^{k}}\right\|_{\mathcal{A}}^{\varepsilon_{k}} \\
& \leq \prod_{k=0}^{\infty}\left(\frac{\|c\|_{\mathcal{A}}}{\|c\|_{\mathcal{B}}}(2 C)^{k}\|c\|_{\mathcal{B}}^{2^{k}}\right)^{\varepsilon_{k}} .
\end{aligned}
$$


By summing up, we obtain

$$
\begin{aligned}
\sum_{n=0}^{\infty}\left\|c^{n}\right\|_{\mathcal{A}} & \leq \sum_{\varepsilon \in \mathcal{F}} \prod_{k=0}^{\infty}\left(\frac{\|c\|_{\mathcal{A}}}{\|c\|_{\mathcal{B}}}(2 C)^{k}\|c\|_{\mathcal{B}}^{2^{k}}\right)^{\varepsilon_{k}} \\
& =\prod_{k=0}^{\infty}\left(1+\frac{\|c\|_{\mathcal{A}}}{\|c\|_{\mathcal{B}}}(2 C)^{k}\|c\|_{\mathcal{B}}^{2^{k}}\right) .
\end{aligned}
$$

We note that this product converges, if and only if

$$
\sum_{k=0}^{\infty}(2 C)^{k}\|c\|_{\mathcal{B}}^{2^{k}}<\infty
$$

which is the case, if and only if $\|c\|_{\mathcal{B}}<1$.

Now assume that $a \in \mathcal{A}$ is invertible in $\mathcal{B}$ and set $b=\frac{a^{*} a}{\left\|a^{*} a\right\|_{\mathcal{B}}}$. Then $b$ is hermitean, invertible, and $\|b\|_{\mathcal{B}}=1$ and the spectrum $\sigma_{\mathcal{B}}(b)$ is contained in $(0,1]$. Consequently, the spectrum of $c=e-b$ is contained in an interval $\sigma_{\mathcal{B}}(e-b) \subseteq[0,1-\varepsilon] \subseteq[0,1)$, and in particular, $\|e-b\|_{\mathcal{B}}=1-\varepsilon<1$. This implies that

$$
b^{-1}=\sum_{k=0}^{\infty}(e-b)^{k}=\sum_{k=0}^{\infty} c^{k}
$$

with convergence in $\mathcal{B}$.

Consequently, the inverse of $a$ is given by

$$
a^{-1}=\frac{b^{-1} a^{*}}{\left\|a^{*} a\right\|_{\mathcal{B}}} .
$$

We now apply the estimate (16) to the element $c=e-b$ and obtain a first estimate for the norm of $a^{-1}$ in $\mathcal{A}$.

$$
\begin{aligned}
\left\|a^{-1}\right\|_{\mathcal{A}} & \leq \frac{\left\|a^{*}\right\|_{\mathcal{A}}}{\left\|a^{*} a\right\|_{\mathcal{B}}}\left(\sum_{n=0}^{\infty}\left\|c^{n}\right\|_{\mathcal{A}}\right) \\
& \leq \frac{\left\|a^{*}\right\|_{\mathcal{A}}}{\left\|a^{*} a\right\|_{\mathcal{B}}} \prod_{k=0}^{\infty}\left(1+\frac{\|c\|_{\mathcal{A}}}{\|c\|_{\mathcal{B}}}(2 C)^{k}\|c\|_{\mathcal{B}}^{2^{k}}\right) .
\end{aligned}
$$

Finally we estimate the norm of $c=e-\frac{a^{*} a}{\left\|a^{*} a\right\|_{\mathcal{B}}}$ directly by the norms of $a$ and $a^{-1}$. Clearly

$$
\|e-b\|_{\mathcal{A}} \leq\|e\|_{\mathcal{A}}+\|b\|_{\mathcal{A}} \leq 1+\frac{\left\|a^{*} a\right\|_{\mathcal{A}}}{\left\|a^{*} a\right\|_{\mathcal{B}}} \leq 1+\frac{\|a\|_{\mathcal{A}}^{2}}{\|a\|_{\mathcal{B}}^{2}} \leq 2 \frac{\|a\|_{\mathcal{A}}^{2}}{\|a\|_{\mathcal{B}}^{2}} .
$$

On the other hand, since $\mathcal{B}$ is a $C^{*}$-algebra and $a^{*} a$ is positive, we have $\left\|\left(a^{*} a\right)^{-1}\right\|_{\mathcal{B}}^{-1}=$ $\min \left\{\lambda: \lambda \in \sigma\left(a^{*} a\right)\right\}=\lambda_{\min }$. Consequently,

$$
\begin{aligned}
\|e-b\|_{\mathcal{B}} & =1-\frac{\lambda_{\min }}{\left\|a^{*} a\right\|_{\mathcal{B}}} \\
& =1-\frac{1}{\left\|\left(a^{*} a\right)^{-1}\right\|_{\mathcal{B}}\left\|a^{*} a\right\|_{\mathcal{B}}}=1-\frac{1}{\left\|a^{-1}\right\|_{\mathcal{B}}^{2}\|a\|_{\mathcal{B}}^{2}} .
\end{aligned}
$$

Inserting these estimates in (16), we obtain the norm control stated in (11). 
Remarks. 1. The proof is a modification of an approach of Sun [33], the important estimate (13) was derived earlier by Blackadar and Cuntz [7, Lemma 3.8] and Kissin and Shulman [24]. Our main contribution is the representation of the geometric series in (16) as an infinite product, which is amenable to a reasonable asymptotic analysis.

2. As $\left\|a^{*} a\right\|_{\mathcal{A}} \leq 2 C\|a\|_{\mathcal{A}}\|a\|_{\mathcal{B}}$, we can replace the inequality (17) by

$$
\|e-b\|_{\mathcal{A}} \leq(1+2 C) \frac{\|a\|_{\mathcal{A}}}{\|a\|_{\mathcal{B}}}
$$

If $\|a\|_{\mathcal{A}} /\|a\|_{\mathcal{B}} \geq 1 / 2+C$, one obtains a slightly better estimate in (11).

3.2. Application: General Approximation Spaces. In approximation theory differential (semi-) norms appear naturally in the definition of approximation spaces [11].

An approximation scheme on the Banach algebra $\mathcal{A}$ is a family $\left(X_{n}\right)_{n \in \mathbb{N}_{0}}$ of closed subspaces of $\mathcal{A}$ that satisfy $X_{0}=\{0\}, X_{n} \subseteq X_{m}$ for $n \leq m$, and $X_{n} \cdot X_{m} \subseteq X_{n+m}, n, m \in$ $\mathbb{N}_{0}$. If $\mathcal{A}$ is a $*$-algebra, we assume that $e \in X_{1}$ and $X_{n}=X_{n}^{*}$ for all $n \in \mathbb{N}_{0}$. The $n$-th approximation error of $a \in \mathcal{A}$ by $X_{n}$ is $E_{n}(a)=\inf _{x \in X_{n}}\|a-x\|_{\mathcal{A}}$. For $1 \leq p<\infty$ and $w$ a weight on $\mathbb{N}_{0}$ the approximation space $\mathcal{E}_{w}^{p}(\mathcal{A})$ consists of all $a \in \mathcal{A}$ for which the norm

$$
\|a\|_{\mathcal{E}_{w}^{p}}=\left(\sum_{k=0}^{\infty} E_{k}(a)^{p} w(k)^{p}\right)^{1 / p}
$$

is finite (with the standard modification for $p=\infty$ ).

Algebra properties of approximation spaces are discussed in [1,17]. The invertibility of elements in an approximation space was investigated in [17]. In particular, we proved the following result.

Proposition 3.4. If $\mathcal{A}$ is a symmetric Banach algebra with approximation scheme $\left(X_{n}\right)_{n \in \mathbb{N}_{0}}$ and $w$ is a subadditive weight function on $\mathbb{N}_{0}$, then $\mathcal{E}_{w}^{p}(\mathcal{A})$ is inverse-closed in $\mathcal{A}$.

The decisive inequality in the proof of this proposition was

$$
\|a b\|_{\mathcal{E}_{w}^{p}(\mathcal{A})} \leq C_{w}\left(\|a\|_{\mathcal{A}}\|b\|_{\mathcal{E}_{r}^{p}(\mathcal{A})}+\|b\|_{\mathcal{A}}\|a\|_{\mathcal{E}_{r}^{p}(\mathcal{A})}\right) .
$$

In other words, the norm of $\mathcal{E}_{w}^{p}(\mathcal{A})$ is a differential norm in $\mathcal{A}$. (In [17] Proposition 3.4 was stated only for polynomial weights $v(k)=k^{r}$, the statement and proof is identical for general subadditive weights.)

As a consequence of Theorem 3.3 we obtain the following statement, which came as a surprise to us.

Corollary 3.5. Assume that $\mathcal{A}$ is a $C^{*}$-algebra with an approximation scheme $\left(X_{n}\right)_{n \in \mathbb{N}_{0}}$ and that $w$ is a subadditive weight on $\mathbb{N}_{0}$. Then the approximation algebra $\mathcal{E}_{w}^{p}(\mathcal{A})$ admits norm-controlled inversion.

Remarks. (1) In approximation theory one uses the polynomial weights $w(k)=v_{r}(k)=$ $k^{r}$ for $r>0$. In the literature, the resulting approximation space $\mathcal{E}_{v_{r-1 / p}}^{p}(\mathcal{A})$ is usually denoted as $\mathcal{E}_{r}^{p}(\mathcal{A})$, in contrast to our notation.

(2) In many situations more structure is available, e.g., a derivation or an automorphism group on $\mathcal{B}$, and the approximation space $\mathcal{E}_{r}^{p}(\mathcal{A})$ can be identified with a Besov space or some other space, see $[11,25,26]$. 
In this case the estimates for norm control can be improved significantly, even without the assumption that the ambient algebra is a $C^{*}$-algebra. This will be the topic of Part II [18].

\section{ASYMPTOTICS OF NORM-CONTROLLED INVERSION IN DIFFERENTIAL SUBALGEBRAS}

In principle, the estimate in (11) yields a norm-controlling funcion $h$, but the expression is too cumbersome to decode any useful information. Our main effort is to derive a clean asymptotic expression for the norm controlling function. The following theorem offers a norm controlling function in which all constants are explicit and depend only on the structure parameter of the differential norm.

Theorem 4.1. Assume that $\mathcal{B}$ is a $C^{*}$-algebra and $\mathcal{A} \subseteq \mathcal{B}$ is a differential $*$-subalgebra with a common unit and a differential norm satisfying (9) with the structure constant $u=2 C$.

Set

$$
\begin{aligned}
& K=(\ln 2-1 / 2)^{-1} \approx 5.1774, \\
& \gamma_{1}=e \exp \left(\frac{8 \ln u \ln ^{2}(\ln u)}{\ln ^{2} 2}\right) \quad \text { and } \quad \gamma_{2}=\frac{16 \ln u}{\ln ^{2} 2} \\
& \gamma_{3}=e \exp \left(\frac{8 \ln ^{2} K}{\ln u}\right) \quad \text { and } \quad \gamma_{4}=\frac{4}{\ln u} .
\end{aligned}
$$

If $\kappa(a) \geq 5$, then

$$
\left\|a^{-1}\right\|_{\mathcal{A}} \leq \max \left\{\gamma_{1} \frac{\|a\|_{\mathcal{A}}}{\|a\|_{\mathcal{B}}^{2}} e^{\gamma_{2} \ln ^{2} \kappa(a)^{2}}, \gamma_{3} \frac{\|a\|_{\mathcal{A}}}{\|a\|_{\mathcal{B}}^{2}} e^{\gamma_{4} \ln ^{2}\left(2 u^{16} \frac{\|a\|_{\mathcal{A}}^{2}}{\|a\|_{\mathcal{B}}^{2}}\right)}\right\} .
$$

If $\kappa(a)^{2} \geq(\ln u)^{-1}\left(10\|a\|_{\mathcal{A}}^{2} /\|a\|_{\mathcal{B}}^{2}\right)^{\ln 2 / \ln u}$, then always

$$
\left\|a^{-1}\right\|_{\mathcal{A}} \leq \gamma_{1} \frac{\|a\|_{\mathcal{A}}}{\|a\|_{\mathcal{B}}^{2}} e^{\gamma_{2} \ln ^{2} \kappa(a)^{2}}
$$

The following corollary provides a simpler description and an explicit norm controlling function $h$ in the sense of Definition 2.1.

Corollary 4.2. If $\mathcal{A}$ is a differential $*$-subalgebra of a unital $C^{*}$-algebra $\mathcal{B}$ with a common unit, then there exist constants $C_{1}, C_{2}>0$ such that

$$
\left\|a^{-1}\right\|_{\mathcal{A}} \leq C_{1}\|a\|_{\mathcal{A}}\left\|a^{-1}\right\|_{\mathcal{B}}^{2} e^{C_{2} \ln ^{2}\left(\|a\|_{\mathcal{A}}\left\|a^{-1}\right\|_{\mathcal{B}}\right)}
$$

Thus the controlling function may be taken to be

$$
h(x, y)=C_{1} x y^{2} e^{C_{2} \ln ^{2}(x y)} .
$$

Proof. The norm control estimates in Theorem 4.1 depends on the three parameters $\|a\|_{\mathcal{A}},\|a\|_{\mathcal{B}}$ and $\left\|a^{-1}\right\|_{\mathcal{B}}$. We eliminate $\|a\|_{\mathcal{B}}$ by using the embedding inequality $\|a\|_{\mathcal{B}} \leq$ $\|a\|_{\mathcal{A}}$ and the Banach algebra inequality $\kappa(a)=\|a\|_{\mathcal{B}}\left\|a^{-1}\right\|_{\mathcal{B}} \geq 1$, whence $\|a\|_{\mathcal{B}}^{-1} \leq$ 
$\left\|a^{-1}\right\|_{\mathcal{B}}$. Then both $\kappa(a) \leq\|a\|_{\mathcal{A}}\left\|a^{-1}\right\|_{\mathcal{B}}$ and $\frac{\|a\|_{\mathcal{A}}}{\|a\|_{\mathcal{B}}} \leq\|a\|_{\mathcal{A}}\left\|a^{-1}\right\|_{\mathcal{B}}$. After adapting the constants in (21), we obtain the stated inequality.

Remark. The case distinction in (21) reveals an important and completely new aspect of norm-controlled inversion. The norm of $a^{-1}$ in the smaller algebra $\mathcal{A}$ may depend on both the condition number $\kappa(a)$ in the larger algebra $\mathcal{B}$ and on the embedding parameter $\frac{\|a\|_{\mathcal{A}}}{\|a\|_{\mathcal{B}}}$, whichever number dominates.

In the extreme case of a unitary element in $\mathcal{B}$ we have $a^{-1}=a^{*}$, so that $\kappa(a)=1$ and $\left\|a^{-1}\right\|_{\mathcal{A}}=\|a\|_{\mathcal{A}}$ depends only on the embedding ratio $\frac{\|a\|_{\mathcal{A}}}{\|a\|_{\mathcal{B}}}$.

As a second example we look at the algebra $\mathcal{A}=C^{1}(\mathbb{T})$ of continuously differentiable functions on the torus embedded in $\mathcal{B}=C(\mathbb{T})$. Consider the sequence

$$
a_{n}(t)=1+\frac{1}{2} \cos 2 \pi n t \text {. }
$$

Then $1 / 2 \leq a_{n}(t) \leq 3 / 2$ and $\kappa(a)=3$, whereas

$$
\frac{\left\|a_{n}\right\|_{C^{1}}}{\left\|a_{n}\right\|_{C}}=\frac{3+2 \pi n}{3}
$$

In this case the norm of $a_{n}^{-1}$ in $C^{1}$ is of order

$$
\left\|a_{n}^{-1}\right\|_{C^{1}} \geq 2 \pi n
$$

and is completely determined by the ratio $\frac{\left\|a_{n}\right\|_{C^{1}}}{\left\|a_{n}\right\|_{C}}$ and not by the condition number of $a_{n}$ in $C(\mathbb{T})$.

The remainder of this section is devoted to proving the asymptotics. In view of (11) we introduce the function

$$
f(u, v, c)=\prod_{k=0}^{\infty}\left(1+c u^{k} v^{2^{k}}\right) .
$$

We are interested in the asymptotic behavior of $f$ as $v$ tends to 1 for fixed $u$ and want to express the constants in terms of $u$ as explicitly as possible. Setting

$$
u=2 C, \quad v=1-\frac{1}{\kappa(a)^{2}}, \quad \text { and } c=2 \frac{\|a\|_{\mathcal{A}}^{2}}{\|a\|_{\mathcal{B}}^{2}}\left(1-\frac{1}{\|a\|_{\mathcal{B}}^{2}\left\|a^{-1}\right\|_{\mathcal{B}}^{2}}\right)^{-1}
$$

Theorem 3.3 says that

$$
\left\|a^{-1}\right\|_{\mathcal{A}} \leq \frac{\|a\|_{\mathcal{A}}}{\|a\|_{\mathcal{B}}^{2}} f(u, v, c)
$$

We will prove the following estimate for $f(u, v, c)$.

Proposition 4.3. Assume that $\ln u / \ln v^{-1} \geq 16$ and set $K=(\ln 2-1 / 2)^{-1}$. Then

$$
f(u, v, c) \leq \begin{cases}e \exp \left(\frac{8 \ln u \ln ^{2}(\ln u)}{\ln ^{2} 2}\right) \exp \left(\frac{8 \ln u}{\ln ^{2} 2} \ln ^{2}\left(\frac{1}{\ln v^{-1}}\right)\right) & \text { if } \ln u / \ln v^{-1} \geq(K c)^{\ln 2 / \ln u} \\ e \exp \left(\frac{8 \ln ^{2} K}{\ln u}\right) \exp \left(\frac{4}{\ln u} \ln ^{2} c\right), & \text { if } \ln u / \ln v^{-1}<(K c)^{\ln 2 / \ln u} .\end{cases}
$$


Proof of Theorem 4.1. Our main theorem follows from Proposition 4.3 by substitution. We keep in mind that $u=2 C \geq 2$ is the structure constant of the differential algebra and $v=1-\kappa(a)^{-2}<1$. In particular the principal parameter $\left(\ln v^{-1}\right)^{-1}$ satisfies

$$
\frac{1}{\ln v^{-1}}=-\frac{1}{\ln \left(1-\kappa(a)^{-2}\right)} \leq \kappa(a)^{2}, \text { for all } \kappa(a) \geq 1,
$$

and $\lim _{\kappa(a) \rightarrow \infty}-\ln \left(1-\kappa(a)^{-2}\right) \kappa(a)^{2}=1$. The restriction $\kappa(a) \geq 5$ comes from the assumption $\ln u / \ln v^{-1} \geq 16$ in Proposition 4.3. Solving for $\kappa(a)$ yields

$$
\kappa(a) \geq\left(1-u^{-1 / 16}\right)^{-1 / 2} \approx 4.857
$$

and since $u=2 C \geq 2$, we may take $\kappa(a) \geq 5>\left(1-2^{-1 / 16}\right)^{-1 / 2}$.

Note that

$$
c=2 \frac{\|a\|_{\mathcal{A}}^{2}}{\|a\|_{\mathcal{B}}^{2}} \frac{1}{1-\kappa(a)^{-2}} \geq 1
$$

depends on $a$, but not on the index $k$ in the infinite product (22).

The restriction for the special case follows from making the condition $\ln u / \ln v^{-1} \geq$ $(K c)^{\ln 2 / \ln u}$ in (23) explicit. We have

$$
\kappa(a)^{2} \ln u \geq \frac{\ln u}{\ln v^{-1}} \geq\left(2 K \frac{\|a\|_{\mathcal{A}}^{2}}{\|a\|_{\mathcal{B}}^{2}} \frac{1}{1-\kappa(a)^{-2}}\right)^{\ln 2 / \ln u} \geq\left(10 \frac{\|a\|_{\mathcal{A}}^{2}}{\|a\|_{\mathcal{B}}^{2}}\right)^{\ln 2 / \ln u}
$$

We carry out the proof of Proposition 4.3 in several steps with intermediate lemmas.

Step 1. Determine the maximum of $1+c u^{k} v^{2^{k}}$.

The asymptotics of $f(u, v, c)$ for $v \rightarrow 1$ will depend on the value of $u$. In the following we will often need the logarithmic ratio of $u$ and $v$, and so we define the number $\xi$ by

$$
2^{\xi}=\frac{\ln u}{\ln v^{-1}} \quad \text { and } \xi=\log _{2}\left(\frac{\ln u}{\ln v^{-1}}\right),
$$

where $\log _{2} z=\ln z / \ln 2$ is the $\log$ arithm with basis 2 . We will need $\xi \geq 4$ and thus $2^{\xi} \geq 16$ in the proof. Note that since $u=2 C \geq 2$ and $v<1, \xi \geq 4$ is well-defined and that $\lim _{v \rightarrow 1-} \xi=\infty$. Furthermore

$$
v^{2^{\xi}}=\exp \left(\ln v \frac{\ln u}{\ln v^{-1}}\right)=u^{-1} .
$$

Lemma 4.4. If $u \geq 1$ and $v<1$, then

$$
1+c u^{k} v^{2^{k}} \leq 1+c u^{\xi} \quad \forall k \in \mathbb{N} .
$$

Proof. Let $c_{k}=c u^{k} v^{2^{k}}$. Then

$$
\frac{c_{k+1}}{c_{k}}=\frac{c u^{k+1} v^{2^{k+1}}}{c u^{k} v^{2^{k}}}=u v^{2^{k}}
$$

and $1+c_{k}$ is decreasing, if and only if $u v^{2^{k}} \leq 1$. Thus $1+c_{k}$ is decreasing, if and only if $2^{k} \geq \frac{\ln u}{\ln v^{-1}}=2^{\xi}$. Set $K=\lceil\xi\rceil$. Then $1+c_{k}$ is decreasing for $k \geq K=\lceil\xi\rceil$, increasing 
for $k<K$, and the maximum of $c_{k}$ is taken at $K$. Writing $K=\lceil\xi\rceil=\xi+\eta$ for some $\eta \in[0,1)$ and using (26), we find that

$$
\begin{aligned}
u^{K} v^{2^{K}} & =u^{\xi+\eta}\left(v^{2^{\xi}}\right)^{2^{\eta}} \\
& =u^{\xi+\eta-2^{\eta}} \leq u^{\xi}
\end{aligned}
$$

as claimed. (In fact $u^{\xi+\eta-2^{\eta}} \leq u^{\xi-0.91}$.)

Step 2. Split the infinite product (22) into a finite product $\prod_{k=0}^{M}$ and a remainder $\prod_{k=M+1}^{\infty}$ and estimate the remainder first.

We first determine a cut-off index $M$, so that $\prod_{k=M+1}^{\infty}\left(1+c u^{k} v^{2^{k}}\right) \leq e$.

Lemma 4.5. Let $K=\frac{1}{\ln 2-1 / 2}$ and choose $M$, such that

$$
M+1 \leq \xi+2 \ln _{2}\left(\max \left(\xi, \frac{\ln (K c)}{\ln u}\right)\right) \leq M+2 .
$$

Then

$$
\prod_{k=M+1}^{\infty}\left(1+c u^{k} v^{2^{k}}\right) \leq e
$$

Proof. Using the estimate

$$
1+c u^{k} v^{2^{k}} \leq \exp \left(c u^{k} v^{2^{k}}\right)
$$

the remainder term is bounded by

$$
\prod_{k=M+1}^{\infty}\left(1+c u^{k} v^{2^{k}}\right) \leq \exp \left(\sum_{k=M+1}^{\infty} c u^{k} v^{2^{k}}\right) .
$$

By Step 1 the sequence $k \rightarrow u^{k} v^{2^{k}}$ is decreasing for $k \geq M \geq \xi$, and we estimate the occurring sum by the integral

$$
\sum_{k=M+1}^{\infty} u^{k} v^{2^{k}} \leq \int_{M}^{\infty} u^{x} v^{2^{x}} d x
$$

After the substitution $y=2^{x} \ln v^{-1}$ ( precise details: $x=\log _{2}\left(y / \ln v^{-1}\right)$ and $d x=\frac{1}{\ln 2} \frac{d y}{y}$, $u^{x}=\left(\frac{y}{\ln v^{-1}}\right)^{\log _{2} u}$ ) the integral turns into

$$
\int_{M}^{\infty} u^{x} v^{2^{x}} d x=\frac{1}{\ln 2} \frac{1}{\left(\ln v^{-1}\right)^{\log _{2} u}} \int_{2^{M} \ln v^{-1}}^{\infty} y^{\log _{2} u} e^{-y} d y
$$

The new integral is an incomplete Gamma-function defined as

$$
\Gamma(a, x)=\int_{x}^{\infty} t^{a-1} e^{-t} d t=x^{a-1} e^{-x}\left(1+\sum_{j=1}^{n-1} \frac{(a-1)(a-2) \ldots(a-j)}{x^{j}}+R_{n}(x, a)\right) .
$$

For $n \geq a-1$ the remainder $R_{n}$ satisfies the estimate $R_{n}(x, a) \leq \frac{|(a-1) \ldots(a-n)|}{x^{n}}$. See [12, $8.11(i)]$ or verify directly with integration by parts. 
In our case $a=\log _{2} u+1$ and $x=2^{M} \ln v^{-1} \geq 2 \cdot 2^{\xi} \ln v^{-1}=2 \ln u$. If we choose $M \geq \xi+1$ and $n$ such that $\mathrm{d} a-1 \leq n<2 a+1$, then

$$
0 \leq \frac{|a-j|}{x} \leq \frac{a-1}{x} \leq \frac{\log _{2} u}{2^{M} \ln v^{-1}} \leq \frac{1}{2 \ln 2}=\frac{1}{\ln 4}<1
$$

and consequently

$$
1+\sum_{j=1}^{n-1} \frac{(a-1)(a-2) \ldots(a-j)}{x^{j}}+R_{n}(x, a) \leq 1+\sum_{j=1}^{n} \frac{1}{(\ln 4)^{j}} \leq \frac{1}{1-(\ln 4)^{-1}},
$$

which is a bound independent of $x$ and $a$ and $n$.

Combining these estimates, we find that

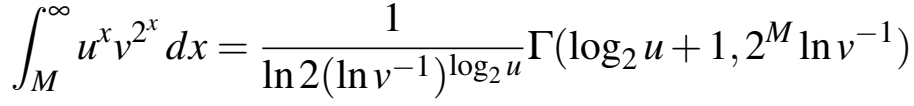

$$
\begin{aligned}
& \leq \frac{1}{\ln 2\left(1-(\ln 4)^{-1}\right)} \frac{\left(2^{M} \ln v^{-1}\right)^{\log _{2} u}}{\left(\ln v^{-1}\right)^{\log _{2} u}} \exp \left(-2^{M} \ln v^{-1}\right) \\
& =K u^{M} \exp \left(-2^{M} \ln v^{-1}\right) \text {. }
\end{aligned}
$$

Here we set $K=\left(\ln 2\left(1-(\ln 4)^{-1}\right)\right)^{-1}=(\ln 2-1 / 2)^{-1}=5.177>1$. Now we choose $M \geq \xi+1$ so that

$$
K c u^{M} \exp \left(-2^{M} \ln v^{-1}\right) \leq 1
$$

Then

$$
\prod_{k=M+1}^{\infty}\left(1+c u^{k} v^{2^{k}}\right) \leq \exp \left(\sum_{k=M+1}^{\infty} c u^{k} v^{2^{k}}\right) \leq \exp \left(K c u^{M} \exp \left(-2^{M} \ln v^{-1}\right)\right) \leq e .
$$

For an explicit estimate of $M$ we note that (30) is equivalent to the estimate

$$
2^{M} \ln v^{-1}-M \ln u \geq \ln (K c) .
$$

By writing $M=\xi+\lambda$ and using $2^{\xi}=\ln u / \ln v^{-1}$, we obtain that

$$
2^{\lambda}-\lambda \geq \xi+\frac{\ln (K c)}{\ln u}
$$

Again since $\xi \geq 4$, we may use

$$
2^{\lambda}-\lambda \geq 2^{\frac{\lambda}{2}+1} \geq 2 \max \left(\xi, \frac{\ln (K c)}{\ln u}\right)
$$

and we obtain that $\lambda \geq 2 \log _{2} \max \left(\xi, \frac{\ln (K c)}{\ln u}\right)$. We find that

$$
M+1 \leq \xi+2 \log _{2}\left(\max \left(\xi, \frac{\ln (K c)}{\ln u}\right)\right) \leq M+2
$$

is a workable choice for $M$. 
Step 3. Estimate the finite part of $\prod_{k=0}^{M}$. We use Lemma 4.4 and the obvious estimate

$$
\prod_{k=0}^{M}\left(1+c u^{k} v^{2^{k}}\right) \leq \max _{k=0, \ldots, M}\left(1+c u^{k} v^{2^{k}}\right)^{M+1} \leq\left(1+c u^{\xi}\right)^{M+1} .
$$

Since the definition of $M$ contains a max, we distinguish two cases.

Case 1. If $\xi \geq \frac{\ln (K c)}{\ln u}$ or, equivalently, $K c \leq u^{\xi}$, then

$$
M+1 \leq \xi+2 \log _{2} \xi \leq 2 \xi
$$

and, since $\xi \geq 4, c \geq 1$, and $u \geq 2$,

$$
\frac{M+1}{c u^{\xi}} \leq \frac{2 \xi}{c u^{\xi}} \leq 1
$$

Then

$$
\left(1+\frac{1}{c u^{\xi}}\right)^{M+1} \leq \exp \left(\frac{M+1}{c u^{\xi}}\right) \leq \exp (1),
$$

and, since $K \geq 1$ and $\ln ^{2}(a / b) \leq 2\left(\ln ^{2} a+\ln ^{2} b^{-1}\right)$,

$$
\begin{aligned}
\left(c u^{\xi}\right)^{M+1} & \leq\left(\frac{u^{2 \xi}}{K}\right)^{M+1} \leq u^{4 \xi^{2}} \\
& \leq \exp \left(4 \frac{\ln u}{\ln ^{2} 2} \ln ^{2}\left(\frac{\ln u}{\ln v^{-1}}\right)\right) \\
& \leq \exp \left(\frac{8 \ln u \ln ^{2}(\ln u)}{\ln ^{2} 2}\right) \exp \left(\frac{8 \ln u}{\ln ^{2} 2} \ln ^{2}\left(\frac{1}{\ln v^{-1}}\right)\right) .
\end{aligned}
$$

Thus the final estimate is

$$
\left(1+c u^{\xi}\right)^{M+1}=\left(1+\frac{1}{c u^{\xi}}\right)^{M+1}\left(c u^{\xi}\right)^{M+1} \leq C(u) \exp \left(\frac{8 \ln u}{\ln ^{2} 2} \ln ^{2}\left(\frac{1}{\ln v^{-1}}\right)\right)
$$

with the constant $C(u)=e \exp \left(\frac{8 \ln u \ln ^{2}(\ln u)}{\ln ^{2} 2}\right)$ that depends only on $u$.

Case 2. If $\xi \leq \frac{\ln (K c)}{\ln u}$ or equivalently, $K c \geq u^{\xi}$, then

$$
M+1 \leq \xi+2 \log _{2} \frac{\ln (K c)}{\ln u} \leq 2 \frac{\ln (K c)}{\ln u} .
$$

In this case we obtain similarly

$$
\begin{aligned}
\left(1+c u^{\xi}\right)^{M+1} & \leq\left(1+K c^{2}\right)^{M+1} \leq\left(K c^{2}\right)^{2 \frac{\ln (K c)}{\ln u}}\left(1+\frac{1}{K c^{2}}\right)^{2 \frac{\ln (K c)}{\ln u}} \\
& \leq e \exp \left(\ln \left(K c^{2}\right) 2 \frac{\ln (K c)}{\ln u}\right) \\
& \leq e \exp \left(\frac{2}{\ln u} \ln ^{2}\left(K c^{2}\right)\right) \\
& \leq C^{\prime}(u) \exp \left(\frac{8}{\ln u} \ln ^{2} c\right)
\end{aligned}
$$

with a constant $C^{\prime}(u)=e \exp \left(\frac{4 \ln ^{2} K}{\ln u}\right)$.

The proof of Proposition 4.3 is now finished. 
Remark. The proof shows that the asymptotic estimates in Theorem 4.1 are in fact

$$
\left\|a^{-1}\right\|_{\mathcal{A}}=o\left(\frac{\|a\|_{\mathcal{A}}}{\|a\|_{\mathcal{B}}^{2}} e^{\gamma_{2} \ln ^{2} \kappa(a)^{2}}\right) \quad \text { for } \kappa(a) \rightarrow \infty .
$$

In fact, in (32) we have given away a factor

$$
K^{-M-1} \leq K^{-\xi} \leq K^{-2 \ln \kappa(a) \ln \ln u} .
$$

Taking this factor into account, we obtain the slightly stronger statement of (33).

\section{Related Concepts of Norm Control And LACK OF Norm CONTROL}

In our definition of norm-controlled inversion we have used only the two quantities $\|a\|_{\mathcal{A}}$ and $\left\|a^{-1}\right\|_{\mathcal{B}}$ to control $\left\|a^{-1}\right\|_{\mathcal{A}}$. By adding more parameters, one is lead to alternative concepts of norm-controlled inversion.

(i) Sun $[33,34]$ considers a nested pair of Banach algebras $\mathcal{A} \subseteq \mathcal{B}$ with a modified differential norm satisfying

$$
\left\|a^{2}\right\|_{\mathcal{A}} \leq 2 C\|a\|_{\mathcal{A}}^{1+\theta}\|a\|_{\mathcal{B}}^{1-\theta}
$$

for a fixed exponent $\theta, 0<\theta<1$. Versions of Lemma 3.2 and Theorem 3.3 hold for such norms. In fact, our proof is a modification of [33]. Probably the asymptotic estimates of Theorem 1.1 could also be adapted to this case. Norms satisfying (34) are important in the study of algebras of infinite matrices with off-diagonal decay.

(ii) In algebras with an approximation scheme one may also include an approximation parameter to control the norm $\left\|a^{-1}\right\|_{\mathcal{A}}$. This fundamental idea is used first in the deep work of Baskakov on the inversion of matrices with off-diagonal decay [4]. To give an explicit example we formulate Theorem 4 of [4] for the Wiener algebra $\mathcal{A}(\mathbb{T})$. Let $a(t)=\sum_{k \in \mathbb{Z}} a_{k} e^{2 \pi i k t}$ with norm $\|a\|_{\mathcal{A}}=\sum_{k}\left|a_{k}\right|$ and define the tail function of $a$ by

$$
\psi_{a}(k)=\sum_{|j| \geq k}\left|a_{j}\right|
$$

If $a \in \mathcal{A}$ and $a(t) \neq 0$ everywhere, then

$$
\left\|a^{-1}\right\|_{\mathcal{A}} \leq 64\|a\|_{\infty}\left\|a^{-1}\right\|_{\infty}^{2} \psi_{a}\left(\frac{1}{4+32\|a\|_{\infty}\left\|a^{-1}\right\|_{\infty}^{2}}\right) .
$$

In contrast to Definition 2.1 this estimate depends not only on the condition number $\|a\|_{\infty}\left\|a^{-1}\right\|_{\infty}$, but also on the rate of approximation of $a$ by trigonometric polynomials, as expressed by the tail function $\psi$.

Similar estimates can be found in Tao's quantitative version of Wiener's Lemma [36].

Lack of Norm Control. Currently there are extremely few examples of Banach subalgebras without norm control. The fundamental example is the algebra $\mathcal{A}=\mathcal{A}(\mathbb{T})$ of absolutely convergent Fourier series as a subalgebra of the algebra $C(\mathbb{T})$ of continuous functions on the torus [28]. In his investigation of the corona problem Nikolski constructed further and rather sophisticated examples of multiplier algebras that lack norm control [29].

The example $\mathcal{A}(\mathbb{T}) \subseteq C(\mathbb{T})$ can be generalized to group algebras [28, 1.2.3]. Precisely, let $G$ be a discrete group that contains at least one torsion free element. We choose the 
$\ell^{1}$-algebra $\mathcal{A}=\ell^{1}(G)$ with respect to convolution and $\mathcal{B}=C^{*}(G)$ the enveloping $C^{*}$ algebra. Then $\ell^{1}(G)$ always lacks norm control in $C^{*}(G)$. Since $\mathbb{Z}$ is a subgroup of $G$, $\ell^{1}(\mathbb{Z}) \simeq \mathcal{A}(\mathbb{T})$ is a closed subalgebra of $\ell^{1}(G)$. Likewise, $C(\mathbb{T})$ can be identified with a closed subalgebra of $C^{*}(G)$. Consequently the lack of norm control follows immediately from the example $(\mathcal{A}(\mathbb{T}), C(\mathbb{T})$.

\section{REFERENCES}

[1] J. M. Almira and U. Luther. Inverse closedness of approximation algebras. J. Math. Anal. Appl., 314(1):30-44, 2006.

[2] R. Balan, P. G. Casazza, C. Heil, and Z. Landau. Density, overcompleteness, and localization of frames. II. Gabor systems. J. Fourier Anal. Appl., 12(3):309-344, 2006.

[3] A. G. Baskakov. Wiener's theorem and asymptotic estimates for elements of inverse matrices. Funktsional. Anal. i Prilozhen., 24(3):64-65, 1990.

[4] A. G. Baskakov. Estimates for the elements of inverse matrices, and the spectral analysis of linear operators. Izv. Ross. Akad. Nauk Ser. Mat., 61(6):3-26, 1997.

[5] J.-E. Björk. On the spectral radius formula in Banach algebras. Pacific J. Math., 40:279-284, 1972.

[6] B. Blackadar. Operator algebras, volume 122 of Encyclopaedia of Mathematical Sciences. SpringerVerlag, Berlin, 2006. Theory of $C^{*}$-algebras and von Neumann algebras, Operator Algebras and Non-commutative Geometry, III.

[7] B. Blackadar and J. Cuntz. Differential Banach algebra norms and smooth subalgebras of $C^{*}$ algebras. J. Operator Theory, 26(2):255-282, 1991.

[8] L. H. Brandenburg. On identifying the maximal ideals in Banach algebras. J. Math. Anal. Appl., 50:489-510, 1975.

[9] S. Dahlke, M. Fornasier, and K. Gröchenig. Optimal adaptive computations in the Jaffard algebra and localized frames. J. Approx. Theory, 162(1):153-185, 2010.

[10] S. Demko, W. F. Moss, and P. W. Smith. Decay rates for inverses of band matrices. Math. Comp., 43(168):491-499, 1984.

[11] R. A. DeVore and G. G. Lorentz. Constructive approximation, volume 303 of Grundlehren der Mathematischen Wissenschaften [Fundamental Principles of Mathematical Sciences]. Springer-Verlag, Berlin, 1993.

[12] Digital Library of Mathematical Functions. Release date 2011. National Institute of Standards and Technology from http://dlmf.nist.gov/

[13] O. El-Fallah and A. Ezzaaraoui. Majorations uniformes de normes d'inverses dans les algèbres de Beurling. J. London Math. Soc. (2), 65(3):705-719, 2002.

[14] O. Èl'-Falla, N. K. Nikol'skiǐ, and M. Zarrabi. Estimates for resolvents in Beurling-Sobolev algebras. Algebra i Analiz, 10(6):1-92, 1998.

[15] K. Gröchenig. Localization of frames, Banach frames, and the invertibility of the frame operator. J.Fourier Anal. Appl., 10(2), 2004.

[16] K. Gröchenig. Time-frequency analysis of Sjöstrand's class. Revista Mat. Iberoam., 22(2):703-724, 2006.

[17] K. Gröchenig and A. Klotz. Noncommutative approximation: inverse-closed subalgebras and offdiagonal decay of matrices. Constr. Approx., 32(3):429-466, 2010.

[18] K. Gröchenig and A. Klotz. Norm-Controlled Inversion in Smooth Banach Algebras, II. Technical Report, to be submitted.

[19] K. Gröchenig and M. Leinert. Wiener's lemma for twisted convolution and Gabor frames. J. Amer. Math. Soc., 17:1-18, 2004.

[20] K. Gröchenig and M. Leinert. Symmetry and inverse-closedness of matrix algebras and functional calculus for infinite matrices. Trans. Amer. Math. Soc., 358(6):2695-2711 (electronic), 2006.

[21] K. Gröchenig, Z. Rzeszotnik, and T. Strohmer. Convergence analysis of the finite section method and Banach algebras of matrices. Integral Equations Operator Theory, 67(2):183-202, 2010. 
[22] A. Hulanicki. On the spectrum of convolution operators on groups with polynomial growth. Invent. Math., 17:135-142, 1972.

[23] S. Jaffard. Propriétés des matrices "bien localisées" près de leur diagonale et quelques applications. Ann. Inst. H. Poincaré Anal. Non Linéaire, 7(5):461-476, 1990.

[24] E. Kissin and V. S. Shul' man. Differential properties of some dense subalgebras of $C^{*}$-algebras. Proc. Edinburgh Math. Soc. (2), 37(3):399-422, 1994.

[25] A. Klotz. Spectral invariance of Besov-Bessel subalgebras. J. Approx. Theory, 164(2):268-296, 2012.

[26] A. Klotz. Inverse Closed Ultradifferential Subalgebras. Arxiv e-prints, Jan. 2012.

[27] M. Lindner. Infinite matrices and their finite sections. Frontiers in Mathematics. Birkhäuser Verlag, Basel, 2006. An introduction to the limit operator method.

[28] N. Nikolski. In search of the invisible spectrum. Ann. Inst. Fourier (Grenoble), 49(6):1925-1998, 1999.

[29] N. K. Nikolski. The spectral localization property for diagonal operators and semigroups. Algebra $i$ Analiz, 21(6):202-226, 2009.

[30] A. Olofsson An extremeal problem in Banach algebras. Studia Math., 145(3):255-264, 2001.

[31] M. A. Rieffel. Leibniz seminorms for "matrix algebras converge to the sphere". In Quanta of maths, volume 11 of Clay Math. Proc., pages 543-578. Amer. Math. Soc., Providence, RI, 2010.

[32] J. Sjöstrand. Wiener type algebras of pseudodifferential operators. In Séminaire sur les Équations aux Dérivées Partielles, 1994-1995, pages Exp. No. IV, 21. École Polytech., Palaiseau, 1995.

[33] Q. Sun. Wiener's lemma for infinite matrices with polynomial off-diagonal decay. C. R. Math. Acad. Sci. Paris, 340(8):567-570, 2005.

[34] Q. Sun. Wiener's lemma for infinite matrices. Trans. Amer. Math. Soc., 359(7):3099-3123 (electronic), 2007.

[35] Q. Sun. Frames in spaces with finite rate of innovation. Adv. Comput. Math., 28(4):301-329, 2008.

[36] T. Tao. A quantitative proof of Wiener's theorem. http://www.math.ucla.edu/tao/preprints/harmonic.html.

Faculty of Mathematics, University of Vienna, Nordbergstrasse 15, A-1090 ViENNA, AUSTRIA

E-mail address: karlheinz.groechenig@univie.ac.at

Faculty of Mathematics, University of Vienna, Nordbergstrasse 15, A-1090 ViENNA, AUSTRIA

E-mail address: andreas.klotz@univie.ac.at 